\title{
In Reply: Trough Melatonin Levels Have No Physiological or Clinical Relevance
}

\author{
Chieh-Hsin Lin ${ }^{1,2,3}$, Hsien-Yuan Lane ${ }^{2,4,5}$ \\ ${ }^{1}$ Department of Psychiatry, Kaohsiung Chang Gung Memorial Hospital, Chang Gung University College of Medicine, Kaohsiung, ${ }^{2}$ Graduate \\ Institute of Biomedical Sciences, China Medical University, Taichung, ${ }^{3}$ School of Medicine, Chang Gung University, Taoyuan, ${ }^{4}$ Department \\ of Psychiatry and Brain Disease Research Center, China Medical University Hospital, Taichung, ${ }^{5}$ Department of Psychology, College of Medical \\ and Health Sciences, Asia University, Taichung, Taiwan
}

We thank the author(s) of the Letter to Editor for providing valuable comments to our recent study which compared trough melatonin levels in plasma among elderly people with various severities of cognitive deficits [1].

For the first comment, we have listed the sampling time between 8-12 AM as a limitation in our paper [1], while another study has limited the subject selection to those with specimens collected during a similar time period between 7-11 AM [2].

The second comment is about the high melatonin level in our study. While the reference range of melatonin in peripheral blood has been previously reported to be up to $80.4 \mathrm{pg} / \mathrm{ml}$ at $8 \mathrm{Am}$ in heathy adutls [3], we have discussed our result and provided possible reasons [1]. Briefly, potential confounders such as global variation in sunshine duration may have contributed to the finding [1].

The third comment relates to our assay method and its result. We used a human melatonin ELISA kit (MBS704506, https://www.mybiosource.com/human-elisa-kits/melatonin-mt/704506), which has high sensitivity and excellent specificity for detection of human melatonin. However, we agreed with the comment that the possibility of interference was unable to be excluded. We have also regarded the assay using ELISA rather than HPLC as another limitation in our paper [1].

In summary, our study, albeit with several limitations, has been the first one aiming to compare the melatonin

Received: February 13, 2021 / Accepted: February 16, 2021

Address for correspondence: Hsien-Yuan Lane

Department of Psychiatry, China Medical University Hospital, No.

2, Yuh-Der Road, Taichung 404, Taiwan

E-mail: hylane@gmail.com

ORCID: https://orcid.org/0000-0003-2162-8174 levels in different phases of cognitive aging [1]. Further studies are warranted to specifically and feasibly measure melatonin and, if necessary, other related compounds such as its precursors or metabolites, and elucidate their roles in different phases of Alzheimer's disease.

\section{Conflicts of Interest}

No potential conflict of interest relevant to this article was reported.

\section{Author Contributions}

Conceptualization, writing of original draft, review \& editing: Chieh-Hsin Lin, Hsien-Yuan Lane.

\section{ORCID}

Chieh-Hsin Lin ～https://orcid.org/0000-0001-6949-8968

Hsien-Yuan Lane https://orcid.org/0000-0003-2162-8174

\section{REFERENCES}

1. Lin $\mathrm{CH}$, Chiu CC, Lane HY. Trough melatonin levels differ between early and late phases of Alzheimer disease. Clin Psychopharmacol Neurosci 2021;19:135-144.

2. Nogueira LM, Sampson JN, Chu LW, Yu K, Andriole G, Church $\mathrm{T}$, et al. Individual variations in serum melatonin levels through time: implications for epidemiologic studies. PLoS One 2013;8:e83208.

3. Terzieva DD, Mateva ND, Vladimirova-Kitova LG. Melatonin reference limits at 3:00 AM and 8:00 AM in healthy adults. Clin Lab 2009;55:359-361.

(c) This is an Open-Access article distributed under the terms of the Creative Commons Attribution Non-Commercial License (http://creativecommons.org/licenses/by-nc/4.0) which permits unrestricted non-commercial use, distribution, and reproduction in any medium, provided the original work is properly cited. 